\title{
Autonomous Applied Robotics: Ultrasound-Based Robot-Assisted Needle Insertion System Concept and Development
}

\author{
Bence Takács ${ }^{1,2,3}$, Tamás Haidegger ${ }^{1,2,4}$ \\ 1 Antal Bejczy Center for Intelligent Robotics, University Research and Innovation Center (EKIK) \\ 2 John von Neumann Faculty of Informatics, Óbuda University \\ ${ }^{3}$ Doctoral School of Applied Informatics and Applied Mathematics, Óbuda University \\ ${ }^{4}$ Austrian Center for Medical Innovation and Technology (ACMIT), Wiener Neustadt, AT
}

\begin{abstract}
Ultrasound (US) is a popular imaging modality for image-guided minimally invasive surgery (MIS), enabling the faster and more reliable execution of numerous procedures, such as biopsy, electrode placement and vessel cannulation. Blood vessel cannulation is a common, routine intervention, e.g., for blood oxygen level testing. Yet, in particular cases, when the vessel is located deep or veins less stable (with the loss of subcutaneous tissue), it is hard to complete it without US assistance. In this paper, we present a solution for USguided, robot-assisted needle insertion for vein cannulation. We developed an image-guided system to aid needle insertion via active targeting and anatomy-relevant positioning, together with safeguarding features, such as a kinematically enforced Remote Center of Motion (RCM) mechanism. The proposed system comprises a portable US transducer mounted on a KUKA iiwa collaborative robot, a custom designed needle insertion mechanism with adjacent controllers. The US and needle insertion mechanism are attached to the robot through a 3D printed custom designed mounting part with integrated force sensor. The robot arm is responsible for moving the needle to target position with impedance control. The needle insertion mechanism allows the manipulation of the needle along 3 axes. The mechanism was designed for near-surface vein cannulation with an RCM kinematic structure to avoid damage to the vein. The developed system was tested with different types of gelatin phantoms. Vein deformation and tissue motion was examined during US imaging. The control loop of our system is supplemented with vein deformation tissue model and US-based visual servoing.
\end{abstract}

\section{INTRODUCTION}

The application of applied robotic systems has seen a significant rise, even in the traditionally hard domains, such as autonomous vehicles and medical robotics [1]. Besides the collaborative robot systems that appeared on the market, novel sensor technology also helps the implementation of complex systems [2]. In medicine, the wide availability of non-invasive imaging devices has facilitated the development.

Ultrasound (US) is a rather popular imaging technique in field of noninvasive interventions [3]. The advantages over other imaging modalities are :

- The cost of operation is less than other imaging modalities like Magnetic Resonance Imaging (MRI) or Computed Tomography (CT).

- No adverse effects for the human body: The side effect of US is that it can heat up the targeted tissue in Doppler mode.
- Tissue visualization: US can be used to observe tissue movement in real-time.

- Liquid flow visualization: Doppler capable machines can visualize liquid flow for example blood cells in veins.

This possibilities offered by US combined with robotics solutions can be used for many medical intervention, for example vein cannulation, US elastography, tissue sampling, injection treatments. In this field the high accuracy of system is necessary to complete successfully intervention[4]. Today there are lot of portable US devices. Size of this can be smaller than a tablet and the resolution and accuracy is sufficient for clinical grade interventions. Goal of our research was to develop a relatively simple, but stable and accurate system for needle insertion by applying a portable US device and custom designed needle insertion mechanism with a collaborative robot arm. Most of current clinical systems which are applied to needle insertion employ pre-operative images (CT or MRI) to provide robot assisted intervention. This techniques usually need complex registration algorithms and soft tissue can moves between imaging and intervention.

Our first target task is near surface vein cannulation, and in future, we would like to expand capabilities with different biopsy interventions. Near surface vein cannulation is the most common task of anesthesiologists. In most cases, the vein is identified by surface landmarks which are visible or palpable. The vision and tactile information is indispensable for success cannulation. It is routine task but in complex situation when the vein is deeper under skin surface (3-5 mm) the identification is impossible by anesthesiologists and the position and orientation of vein is identified by US imaging. In this research we try to provide an solution for this task. The system is use intra-operative US images for real-time control. This control require algorithm which synchronise motion of robot arm and needle insertion mechanism with US image. It is also an complex task but with real time images the accuracy of the system can be higher.

\section{Review of Past Researches of US-Guided NEEDLE INSERTION}

One of the most rapidly developing field of minimally invasive surgeries is US-based navigation. In robotics USbased surgery are two trend: 
- Hold and operate with a US probe;

- Extending the probe with manipulator or tool guide (for example needle guide).

In the first case, the robotic system is responsible to move the US probe to target position, and hold it during the intervention. In the second case the complete system is working synchronously, and all of the components are registered with each other.

In 2004, a dual armed robotic system was presented [5]. The authors reported a "two-handed" system for surgical context of liver cancer biopsy and ablation. Two robotic arms were used, a LARS robot which developed by Johns Hopkins University (JHU) to manipulate a US probe and a 5 DOF needle driver robot [6]. The needle driver robot consists of two separated mechanism:

- A passive arm to adjust/hold in place the end effector (3 DOF);

- A 2 DOF chain drive robot arm with RCM mechanism. [7]

The LARS robot can make a scan on phantom (on predetermined area), the 2D US images and probe positions are compiled to 3D US images. The 3D Slicer interface was used to identify the target and entry point. The needle driver robot placed the tip of needle to entry point. The insertion is driven manually. They used depth marker on needle and real-time US image in computer display to get desired depth. The needle placement experiments were tested with three different phantoms:

- Overripe plum immersed in a water tank;

- Calf liver with embedded olives (representing some pathologies);

- Mechanical phantom (Cylindrical plate with four different size vertical pins).

With mechanical phantom from 10 trails, the average measured error (distance between needle tip and target in and position) was $3.0 \mathrm{~mm}$. This error is comprised the elements of the system error.

Kojcev et al. in 2016 presented a two arm system for US-guided needle placement [8]. They used the two KUKA LBR iiwa 7 R800 robots, two Intel RealSense F200 RGBD sensors and Ultrasonix SonixTable with C5-2/60 probe. One of the robot is used to move and hold the US probe and the other to perform precise needle placement. The $3 \mathrm{D}$ scanning of the phantom is registered by the region of interest of the RGB-D data, which is selected by operator. The 2D images and robot position is compiled to 3D US data. After scanning the intra-operative images are registered with preoperative images (MRI or CT). After scanning the needle insertion point and trajectory computed. To avoid the collision between needle and US probe they determined 3 zones on surface for injection points:

- Too far from probe: needle will not reach target,

- Possible injection points,

- Too close to probe: robot may collide.

During the insertion, the real-time US image is processed, providing needle and target tracking. The continuous tracking allows to update the needle trajectory with visual servoing.

To validate target tracking accuracy, they used two robots, where one operates the US probe, and the other moved the phantom linearly $( \pm 20 \mathrm{~mm})$, The accuracy based on 9 trials was $0.6 \pm 0.1 \mathrm{~mm}$. The insertion accuracy was measured with water and gelatin phantom with different needle speed and needle angle. The best accuracy was $0.81 \pm 0.19 \mathrm{~mm}$ in gelatin with slow speed $(1 \mathrm{~mm} / \mathrm{s})$ at a steep angle $\left(45^{\circ}\right)$.

Zettinig et al. published an 3D US based visual servoing implementation for neurosurgical interventions [9]. They used an KUKA LBR iiwa 7 R800 robot arm and Ultrasonix Sonix RP system with 4DC7-3/40 Convex 4D transducer. The presented system used pre-operative images (MRI or CT) to determine the target point and the needle path. With real-time Ultrasound $3 \mathrm{D}$ and $2 \mathrm{D}$ images after registration the neurosurgical navigation framework tracks the target. Patient movement was compensated by visual servoing. The visual servoing was combined with force control to dual-control architecture. The robot applied constant contact force between patient (phantom) and US probe. The probe navigation is automated, the needle insertion is driven manually. To hold the needle in right orientation, a needle guide is rigidly attached to the US probe. The insertion depth is verified on the live $2 \mathrm{D}$ image. In order to evaluate the tracking and motion compensation capabilities of system the phantom was mounted for KUKA LBR 4+ robot arm and horizontal sweep of $\pm 60 \mathrm{~mm}$ was acquired. The final visual servoing positioning error was $0.59 \pm 0.33 \mathrm{~mm}$ for translation and $0.61^{\circ} \pm 0.45^{\circ}$ for rotation. The average orthogonal needle positioning error was $1.36 \pm 0.33 \mathrm{~mm}$.

Balter et al. presented an fully automated vein-puncture device. The system used a 3D NIR and US imaging to localize the vein. The robotic part of system have $9 \mathrm{DOF}$ in two separated mechanism:

- 6 DOF Base Positioning System;

- 3 DOF Needle manipulator.

The base positioning system consists of thee translation and tree rotation joints to move the US probe and the needle insertion end-effector unit over to target position. The needle manipulator is a serial kinematic arm with integrated force sensor to detect insertion force. With NIR illumination and stereo vision, the device provide a $3 \mathrm{D}$ map of the veins. A human operator selects the target for cannulation. After selection, the target is tracked in realtime. On US images the needle tip is localized. At the initial step the operator should mark the target vessel center point. After initialization the needle tip and vessel center point is tracked automatically. An axial force sensor is embedded along the needle in manipulator to detect when the needle punctures the skin and vessel wall. The system was tested in different scenarios. Over free spacing position trials, where a cylindrical calibration platform was used to measure the accuracy and repeatability of the robot in the work space. The 3-D positioning error was $0.22 \pm 0.05 \mathrm{~mm}$, repeatability error was $0.03 \mathrm{~mm}$. To evaluate stereo vision accuracy, a tracking experiments were conducted. With the continuously moved cylindrical calibration platform $(10 \mathrm{~mm} / \mathrm{s}$ translation 
and $10 \mathrm{deg} / \mathrm{s}$ rotation speed), the following error was $0.4 \mathrm{~mm}$ and $0.5^{\circ}$.

\section{DEVELOPMENT OF US-BASED NEEDLE INSERTION SYSTEM}

Our research goal was to create a system for robot-assisted US-based vein cannulation. We used different industrial, surgical and custom designed components to realize the complete system. During design we endeavored to construct rigid, high precision components. The target vein dimension was $5 \mathrm{~mm}$. The low accuracy and repeatability error is necessary for successful intervention.

\section{A. System overview}

Fig. 1. shows the overall architecture of our experimental system. This setup of limited robotic assistance translates to a Level of Autonomy 3 robot [10]. System components include:

- A conventional portable 2D ultrasound system (MicrUs EXT-H1, Telemed);

- Kuka iiwa 7 R800 robot arm to move and hold the tools;

- Optoforce OMD-10-SA-10N force sensor to measure real-time contact force;

- 3 DOF custom designed RCM [11] kinematic needle insertion mechanism;

- PC-based workstation to provide control of system and 2D ultrasound processing.

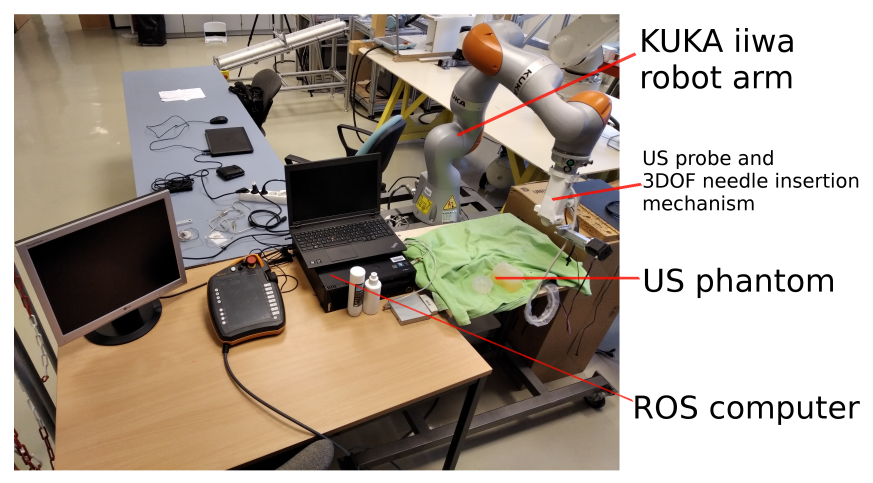

Fig. 1. Robot-assisted US-based vein cannulation system environment. KUKA iiwa collaborative robot arm is used to move the US probe and needle insertion mechanism. Vein and tissue behaviour are examined on US phantom. The US images processing and robot control are executed in ROS.

Our concept was to integrate all tools and sensors to one end-effector. To attach US transducer, force sensor and needle insertion robot we designed an adapter to the flange. The adapter is 3D printed from PLA material. The robot is a KUKA LBR iiwa 7 R800 industrial collaborative robot arm. The first element of whole intervention process is determination of scanning area. In manual drive mode the robot records the contact force and tool location when an operator moves it. With this process the operator select the region for automatic scanning. The KUKA iiwa robot has capability to measure external forces on the flange, but in manual mode, the contact force between the US transducer and phantom is outside of internal force measurement loop. To measure the contact force in real-time we integrated an Optoforce OMD-10-SA-10N to the tool adapter. In automatic scanning the robot applies minimal force (explained below), and verifies the applied force using integrated force sensor, but the force signal for control loop is provided by robot arm. The system control executed over Robot Operating System (ROS https://www.ros.org/). Most of the components are integrated to the ROS platform to provide real-time data forwarding and robot control. We use ROSSmartServo (https: / / github.com/IFL-CAMP / i iwa_ stack/wiki) extension on robot arm to get real-time controllability, sensor information and position data as well.

\section{B. Investigation of Superficial Veins on phantom}

With early prototype, where just US transducer was mounted on robot flange, we investigated the behavior of near surface veins during manual scanning. We used several different ultrasound phantom to test the system with different materials. The first generation was an edible gelatin phantom. It was useful for short-term tests. The objects (tubes and molded cubes from silicon) were clear and recognizable. The gelatin at higher contact force got distorted and melt at higher temperature. The second generation was a wax gel phantom. The quality of the US images were closer to human tissue than with the first generation. The permanent deformation was still significant under high pressure. The first and second generations also had the disadvantage that high contact force the movement of US probe tear the material. To solve this problem we created the third generation US phantom. It made from Polyvinyl Chloride Plastisol (PVCP). Carvalho et al. in 2016 published an PVCP breast phantom for ultrasound investigation. [12] In 2017, Matheo et al. published a comparison of manufacturing protocols of PVCP [13]. We tested both protocols and chose the microwave heating method for our phantom because of the controllability of the manufacturing. We used silicone tubes as substitute for veins.

In the followings, we present the result of the measurement of superficial veins on PVCP based vein phantom. The measurement process was to test two different size of vein (diameter $3 \mathrm{~mm}$ and $7 \mathrm{~mm}$ ). During the tests, the tool was driven manually in cooperative mode to right dept to get the required force. Given the right position, the tool was held by robot in position mode control. In steady state, we measured the diameter of the tube on the US image. In Fig 2 the results of the measurement can be see. The different sizes of tubes have different deformation for contact force. The bigger tube has relative higher deformation then small. Deformations are about $50 \%$ for $7 \mathrm{~mm}$ diameter tube (deformed diameter at maximum force $3.41 \mathrm{~mm}$ ) and about $20 \%$ for $3 \mathrm{~mm}$ diameter tube (deformed diameter at maximum force $2.33 \mathrm{~mm}$ ). This is due to diameter and wall thickness ratio is different. The wall thicknesses are $1 \mathrm{~mm}$ (7 mm diameter) and $0.5 \mathrm{~mm}$ (3 $\mathrm{mm}$ diameter). The test environment can be see on Fig 3 


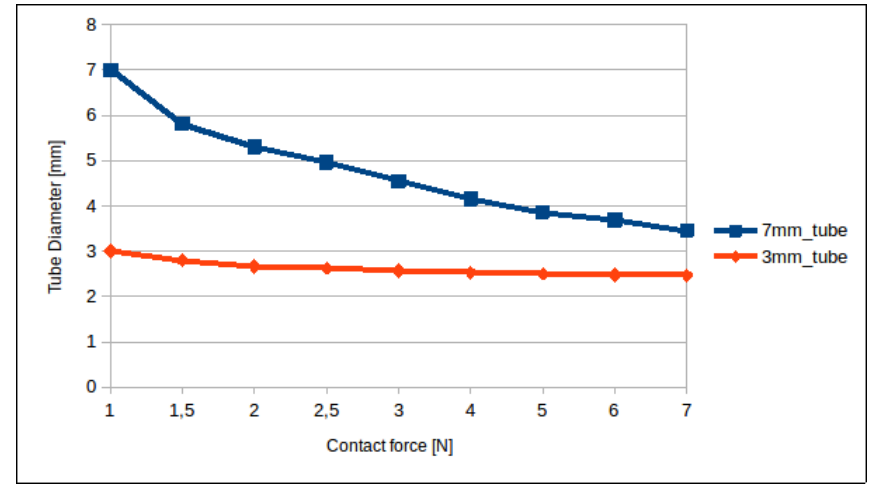

Fig. 2. Measured tube diameters during investigation of near surface veins by robot-held US. Blue: $7 \mathrm{~mm}$ tube Red: $3 \mathrm{~mm}$ tube

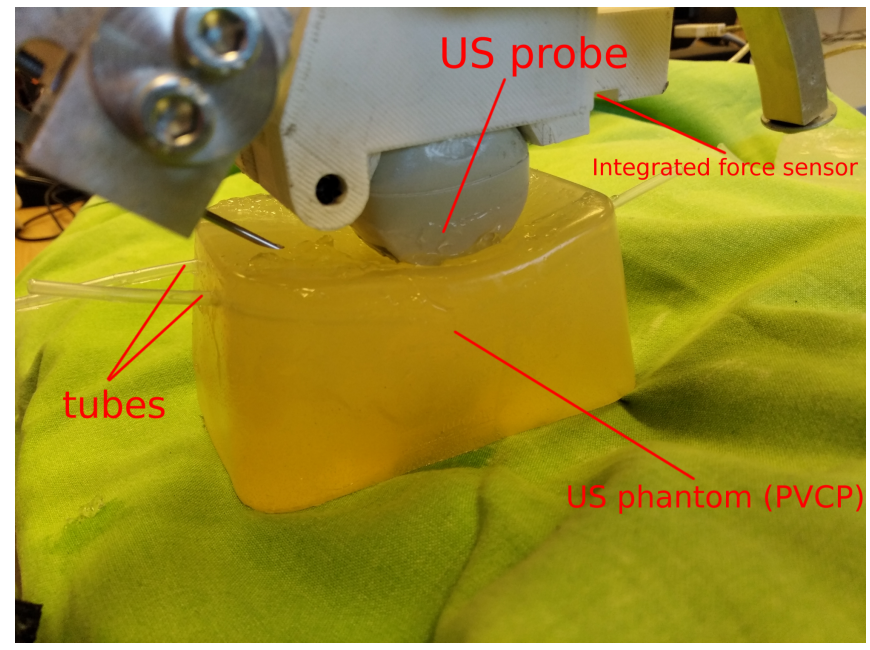

Fig. 3. Test environment with $3 \mathrm{~mm}$ tubes. The US phantom is made from PVCP material. The test is examined with contact forces with two different size of tube.

It was concluded from the data that different maximum contact forces are required for different diameter veins in the phantom. Using the collected data, we designed the control loop of our robotic assisted US based needle insertion system (Fig. 4).

The control loop of the whole system contains the 3D scanning and the US-based needle insertion unit also. Those are different processes, but both of them require the tissue model based contact force control. The process controller module decides which mode is to be used. The desired tool path is generated by the process controller module. This data consists of raw 6 DOF position points. The path data is supplemented with a desired contact force in tissue model module. This is an estimated force, which is enough to minimize the deformation. The difference between tissues and the veins cause a minimal residual deformation. To remove this error, a real-time visual servoing may be required. The tissue model has an input parameter target vein diameter. This parameter is calculated automatically after 3D scanning and vein recognition. During the 3D scanning, the contact force is as small as possible, because in this case the tissue motion and deformation is negligible. During the needle insertion, the puncture force can move the tissue. To minimize this tissue motion it is advisable to use the maximum contact force by the US probe resulting minimal vein deformation. This vertical force helps keep in place the tissue.

Further data sampling for tissue modeling belongs to our future work. The important element of the control loop is the 3D needle insertion mechanism. In the next section we present the designing of this unit.

\section{Designing 3 DOF Needle insertion module}

The Remote Centre of Motion is a wildly used kinematic structure in the field of robotic surgery [?]. Our needle insertion mechanism was also designed based on the RCM, and the longitudinal axis of needle go through the RCM point. The mechanism has 3 active joints. Two of them are translational, and the third is a rotational axis (Fig. 5.).

The first joint is responsible for moving the RCM point vertically. This vertical axis is same with US image vertical axis. The RCM point falls into the same plane of US image. By rotating the second joint, we can choose which radial line (line which starting from RCM point) is to be used to insert the needle to target point. The insertion angle is changed during the intervention because at the entry point the needle has to be at an obtuse angle. This ensures that the needle does not bend. The position and orientation of the vein can be specified following the 3D reconstruction of the US images. Based on this position we can specify the needle's entry point and the target position of the vein center point. By moving the first axis, we can drive the RCM point into the centre of the vein (Fig. 6/a), then the needle is inserted into this point through an obtuse angle(Fig. 6/b). The needle can be pivoted to an acute angle once the tip of it reached the RCM point (Fig. 6/c), then the needle can be pushed forward along the vein to establish a stable fixation of needle in vein (Fig. 6/d)

. Due to the desired mechanical constraints, the mechanism will rotate around the tip of the needle at the RCM point, and it cannot make any damage in the wall of the vein.

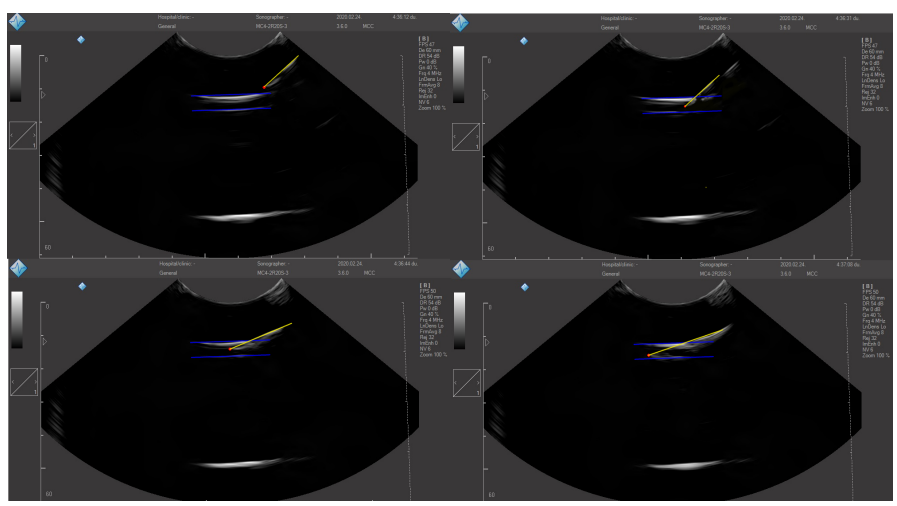

Fig. 6. Needle insertion step by step in US phantom. Red dot marks tip of the needle, blue lines are the tube, yellow line represents the angle of the needle. Figure a: needle insertion in obtuse angle, b: needle tip in RCM point, c: pivoting the needle to acute angle, d: pushing the needle forward along the vein

All axes of the mechanism are driven by stepper motors. 


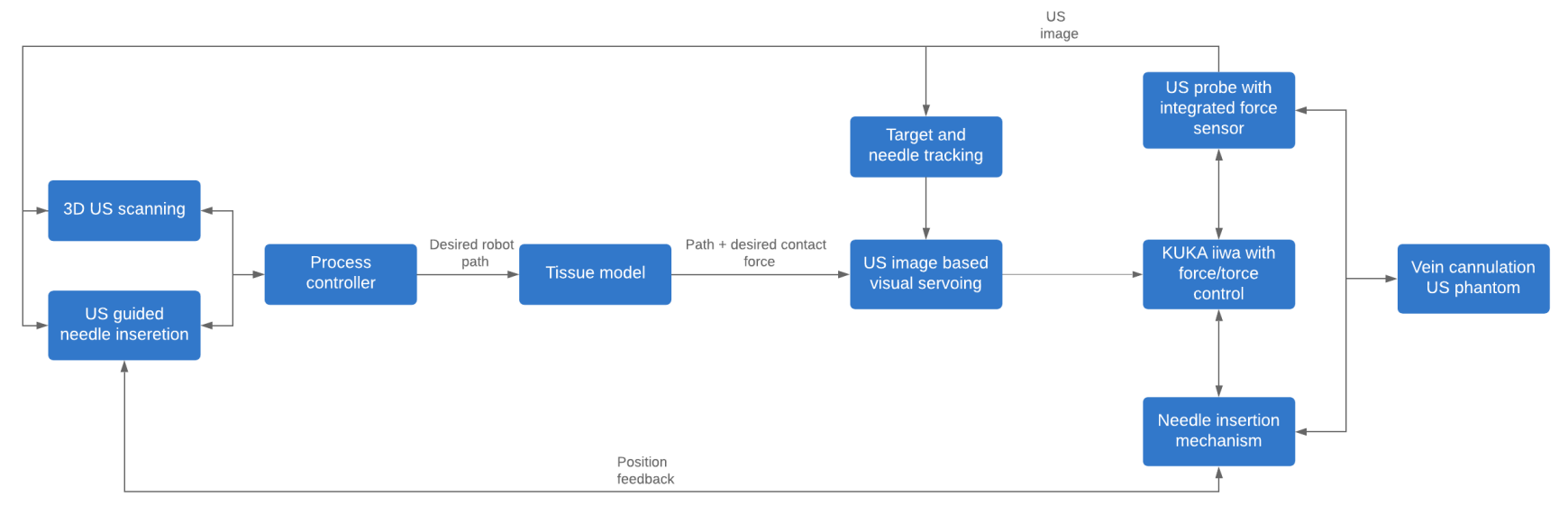

Fig. 4. Control loop of our US-based robot-assisted needle insertion system. The redesigned control loop is complemented with vein deformation tissue model and US-based visual servoing.

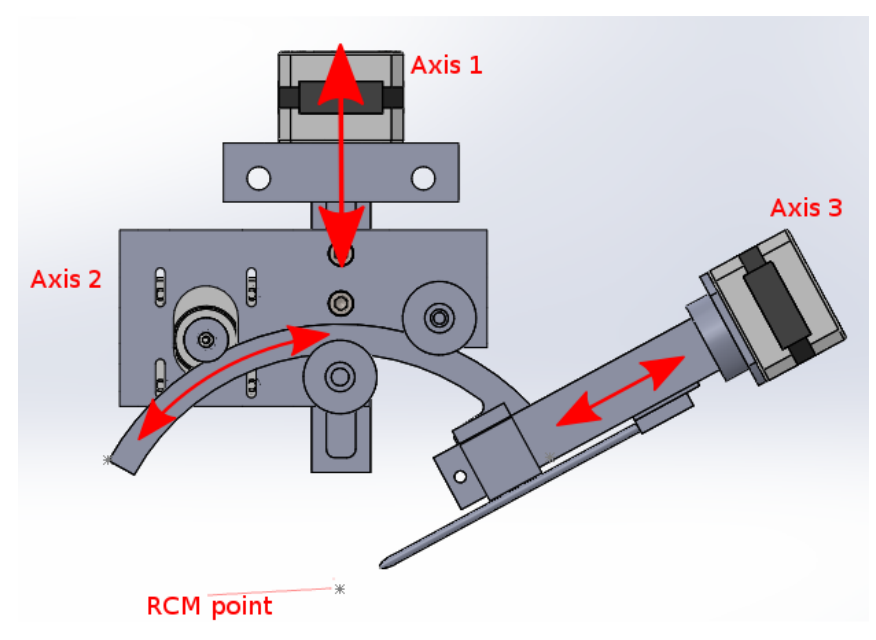

Fig. 5. 3 DOF needle insertion mechanism on CAD model. Axis 1: Moving RCM point in vertical plane. Axis 2: Rotating the end of mechanism around the RCM point. Axis 3: Insert the needle to RCM point

Stepper motor has the advantage that it does not require encoder to measure the position. Each step can be converted to a linear distance or an angular angle. The disadvantage of a simple stepper motor (without encoder) is the possible step loss. Usually, this is caused by the high torque. In our application, the gear ratio is high, so the possibility of step loss is low. As mentioned above, most of the system components are integrated to ROS. To integrate the needle insertion mechanism also to ROS we used an Arduino (https://www.arduino.cc/) based motor controller to drive the all axis. With this, the position data of the mechanism is available in real time for all components. The controlling of the mechanism also is performed from ROS over topics. The mechanism was tested with manual control on US phantom. The manual vein cannulation can be see in Fig 6 During the manual tests in most of cases the vein cannulation was successful. The accuracy and repeatability tests are to follow.

\section{RESUlTS}

We collected data on the behavior of artificial veins under US scanning. From the laboratory experiments, we came to the conclusion that a tissue model is required to apply enough contact force without significant vein deformation. The tissue model uses the real-time US images and force data. The force data has two source, those are force sensor which is integrated to US probe holder and robot arm internal force measuring module. With this model, we redesigned the architecture of our system. The new control loop is representing a model-based approach, supplemented with visual servoing. During visual servoing of needle, the target point of vein and tip of the needle are tracked, and the contact force caused tissue (target vein) motion is compensated in real-time. The determination of desired contact force is performed by the tissue model, and real-time visual servoing of the US probe provides further means to minimize the remaining error of contact force. The implementation of the new control loop on the KUKA iiwa belongs to future work.

After the specification of the system architecture, we designed an 3 DOF needle insertion mechanism. It is designed based on an RCM. The main goal was to access the near surface veins for cannulation. Due to RCM mechanism, in the RCM point the needle can orient without needle tip motion. This mechanical limitation can minimize the chance of vein injury. The mechanism has a relatively big working space, therefore it can be used for various biopsy tasks as well in the future, in this scenario, the needle insertion path can be change without target insertion point movement. The mechanism was tested with manual drive. The needle placement accuracy is measured in water and US phantom. The detailed result will be published in a further publication.

\section{FUTURE WORK}

The US phantom tests directed us towards more complex and tissue-compliant control modes: model-based control and visual servoing may lead to successful intervention. In the future, we are going to work on the implementation an testing 
of those components in the control loop. After that, accuracy and repeatability tests will be conducted to validate the whole system in vitro and ex vivo.

\section{CONCLUSION}

In this paper, we presented the results of an ongoing development towards an ultrasound-based robot-assisted needle insertion system. We collected data from tests on our US vein phantom with the current prototype. Based on the measurements, we created a new control architecture for the system. Tissue model integration and US-based visual servoing are deemed to be required for successful intervention. The required component will be implemented and tested in the near future.

\section{ACKNOWLEDGMENT}

The research presented in this paper was carried out as part of the EFOP-3.6.2-16-2017-00016 project in the framework of the New Széchenyi Plan. B. Takács is supported by the Doctoral School of Applied Informatics and Applied Mathematics Óbuda University. T. Haidegger is supported through the ÚNKP New National Excellence Program of the Ministry of Human Capacities. T. Haidegger is a Bolyai Fellow of the Hungarian Academy of Sciences.

\section{REFERENCES}

[1] D. A. Drexler, A. Takács, T. D. Nagy, and T. Haidegger, "Handover process of autonomous vehicles: Technology and application challenges," Acta Polytechnica Hungarica, vol. 16, no. 9, pp. 235-255, 2019.

[2] A. Takacs, I. Rudas, D. Bösl, and T. Haidegger, "Highly automated vehicles and self-driving cars [industry tutorial]," IEEE Robotics \& Automation Magazine, vol. 25, no. 4, pp. 106-112, 2018.

[3] C. Urbán, P. Galambos, G. Györök, and T. Haidegger, "Simulated medical ultrasound trainers a review of solutions and applications," Acta Polytechnica Hungarica, vol. 15, pp. 111-131, 012018.

[4] T. Haidegger, "Probabilistic method to improve the accuracy of computer-integrated surgical systems," Acta Polytechnica Hungarica, vol. 16, no. 8, pp. 119-140, 2019.

[5] E. M. Boctor, G. Fischer, M. A. Choti, G. Fichtinger, and R. H. Taylor, "A dual-armed robotic system for intraoperative ultrasound guided hepatic ablative therapy: a prospective study," in IEEE International Conference on Robotics and Automation, 2004. Proceedings. ICRA '04. 2004, vol. 3, pp. 2517-2522 Vol.3, April 2004.

[6] R. H. Taylor, J. Funda, B. Eldridge, S. Gomory, K. Gruben, D. LaRose, M. Talamini, L. Kavoussi, and J. Anderson, "A telerobotic assistant for laparoscopic surgery," IEEE Engineering in Medicine and Biology Magazine, vol. 14, pp. 279-288, May 1995.

[7] D. Stoianovici, L. L. Whitcomb, J. H. Anderson, R. H. Taylor, and L. R. Kavoussi, "A modular surgical robotic system for image guided percutaneous procedures," in Medical Image Computing and Computer-Assisted Intervention - MICCAI'98 (W. M. Wells, A. Colchester, and S. Delp, eds.), (Berlin, Heidelberg), pp. 404-410, Springer Berlin Heidelberg, 1998.

[8] R. Kojcev, B. Fuerst, O. Zettinig, J. Fotouhi, S. C. Lee, B. Frisch, R. Taylor, E. Sinibaldi, and N. Navab, "Dual-robot ultrasound-guided needle placement: closing the planning-imaging-action loop," International journal of computer assisted radiology and surgery, vol. 11, 04 2016.

[9] O. Zettinig, B. Frisch, S. Virga, M. Esposito, A. Rienmueller, B. Meyer, C. Hennersperger, Y.-M. Ryang, and N. Navab, "3d ultrasound registration-based visual servoing for neurosurgical navigation," International Journal of Computer Assisted Radiology and Surgery, vol. 12, 022017.

[10] T. Haidegger, "Autonomy for surgical robots: Concepts and paradigms," IEEE Transactions on Medical Robotics and Bionics, vol. 1, no. 2, pp. 65-76, 2019.
[11] H. T. Russell, F. Janez, D. G. David, P. K. John, and A. L. David, "Remote center-of-motion robot for surgery," no. US5397323A, 1992.

[12] I. Carvalho, L. De Matheo, J. F. Costa Júnior, C. Santos, M. Kruger, A. Infantosi, and W. Pereira, "Polyvinyl chloride plastisol breast phantoms for ultrasound imaging," Ultrasonics, vol. 70, 042016.

[13] L. De Matheo, L. Maggi, J. F. Costa Júnior, M. Krüger, and W. Pereira, "Comparison of two ultrasonic phantom manufacturing protocols made of polyvinyl chloride plastisol," 032017. 